\title{
Relationship between malnutrition and possible sarcopenia in the AWGS 2019 consensus affecting mortality in hemodialysis patients: a prospective cohort study
}

Kenichi Kono ${ }^{1 *}$, Yoshifumi Moriyama ${ }^{2}$, Hiroki Yabe ${ }^{3}$, Ayaka Hara ${ }^{4}$, Takeki Ishida ${ }^{1}$, Tetsuya Yamada ${ }^{5}$ and Yusuke Nishida ${ }^{1}$

\begin{abstract}
Background: The first objective of this study was to determine the relationship between muscle strength or physical performance and mortality, and the second objective was to show the relationship of Geriatric Nutritional Risk Index (GNRI) to muscle strength and physical performance decline.
\end{abstract}

Methods: We examined handgrip, the 5-times chair stand test, and GNRI in 635 maintenance hemodialysis patients and followed up for 72 months. Predictors for all-cause death were examined using Kaplan-Meier analysis and Cox proportional analysis. The relationship between possible sarcopenia and nutritional disorder (GNRI) was constructed receiver operating characteristic $(\mathrm{ROC})$ curve. We used the Youden index to determine the optimal cutoff points for GNRI.

Results: The multivariate Cox proportional hazard analysis revealed that the GNRI did not show any significance, although handgrip (HR 3.61,95\% Cl 1.70-7.68, p<0.001) and the 5-times chair stand test (HR 1.71 95\% Cl 1.01-2.90, $p=0.045$ ) were significant predictors for mortality. On the evaluation of possible sarcopenia by handgrip strength, the area under curve (AUC) on ROC curve analysis were 0.68 (95\% Cl 0.64-0.72), and 5-chair stand, the AUC on ROC were 0.55 (95\% Cl 0.51-0.60). The cut-off value for the GNRI discriminating those at possible sarcopenia by handgrip strength based on the Youden index was 91.5.

Conclusions: Our study suggests that the handgrip strength test of the AWGS 2019 sarcopenia consensus was a simple and useful tool to predict mortality in chronic hemodialysis patients. Furthermore, GNRI assessment can be a useful tool for screening before assessing possible sarcopenia when it is difficult to perform SARC-F to all patients.

Keywords: Possible sarcopenia, Handgrip strength, GNRI, Mortality

*Correspondence: kohno1209@gmail.com

1 Department of Physical Therapy, International University of Health and Welfare Graduate School, -3 Kozunomori, Narita, Chiba 286-8686, Japan

Full list of author information is available at the end of the article

\section{Background}

Protein-energy wasting syndrome (PEW), which causes adverse changes in nutrition and body composition, is highly prevalent in patients with chronic kidney disease, especially those undergoing dialysis, and is associated with high morbidity and mortality $[1,2]$. The most important element of PEW in elderly patients with Endstage renal disease is the loss of muscle mass [3], which 
is common to the definition of sarcopenia. Sarcopenia is a progressive and generalized skeletal muscle disorder involving accelerated loss of muscle mass, low strength, and low physical performance [4]. Recently, the number of dialysis patients with elderly sarcopenia has been increasing due to aging, physical inactivity [5], and the presence of geriatric diseases and other comorbidities $[6$, 7]. This sarcopenia in dialysis patients is not a primary with no apparent cause other than aging, but secondary sarcopenia with several other factors as the cause. Therefore, it is clinically important to assess and detect malnutrition and sarcopenia for elderly dialysis patients.

The Asian Working Group for Sarcopenia (AWGS) [8] recommends case-finding to identify early signs of sarcopenia and introduces the concept of "possible sarcopenia" defined by low muscle strength with or without reduced physical performance in primary health care or community preventive service setting. However, the relationship between the reference value of these indexes for possible sarcopenia in AWGS2019 and outcomes such as mortality of dialysis patients has not been fully clarified.

Moreover, since few facilities have a physical therapist who can reliably evaluate the muscle strength and physical performance at an outpatient dialysis clinic, it would be useful to identify a screening index by existing clinical laboratory values that can replace indicators of possible sarcopenia. Recently, the geriatric nutritional risk index (GNRI) is used to assess serum albumin kinetics and physical condition and has been utilized as a nutritional assessment index in Japanese chronic kidney disease (CKD) patients $[9,10]$. GNRI's clinical usefulness for predicting mortality has also been reported $[9,11,12]$. If the GNRI can be used to extract sarcopenia cases, it will be effective in managing dialysis therapy by medical doctors, nurses, and clinical engineers.

Therefore, the first objective of this study was to determine the relationship between muscle strength or physical performance and mortality, and the second was to show the relationship of GNRI to muscle strength and physical performance decline.

\section{Methods}

\section{Study population and design}

This study included clinically stable Japanese outpatients in a multicenter hemodialysis clinic from April 2012 to April 2018. All patients who underwent hemodialysis treatment three times per week were included in the study. According to the Japanese Society for Dialysis Therapy data, this is the most common hemodialysis regimen in Japan. Patients were excluded from this study if they had been hospitalized within the previous 3 months, age $<18$ years, dialysis vintage $<6$ months, and refusal to participate. This study was approved by the Ethical
Committee of the International University of Health and Welfare (Approval number. 17-Io-95).

\section{Demographic and clinical laboratory findings}

Patients' demographics, such as age, dialysis vintage, body mass index (BMI), primary kidney disease, and comorbid conditions, were investigated. Laboratory values of serum albumin, serum hemoglobin, $\mathrm{C}$ reactive protein, serum intact parathyroid hormone, standardized dialysis volume $(\mathrm{Kt} / \mathrm{V})$, and normalized protein catabolic rate (nPCR) were also collected.

\section{Assessment of nutritional status by the GNRI}

The GNRI was calculated using the formula described elsewhere: GNRI $=[1.489 \times$ serum albumin $(g / L)]+[41.7 \times($ bodyweight/ideal bodyweight $)][10,13]$. Ideal body weight was defined as having a BMI value of $22 \mathrm{~kg} / \mathrm{m}^{2}$. Malnutrition was defined as a GNRI $<90$, according to previous studies $[9,14]$.

\section{Measurement of muscle strength and physical performance}

In determining possible secondary sarcopenia, muscle strength and physical performance were assessed with reference to the algorithms for the community in the AWGS 2019 consensus of the sarcopenia criteria. Muscle strength was assessed by handgrip strength to indicate skeletal muscle strength [8], and handgrip strength was measured using a Smedley-spring type dynamometer (101A HATS, Tokyo). The handgrip's low muscle strength diagnostic cut-offs were $<28.0 \mathrm{~kg}$ for men and $<18.0 \mathrm{~kg}$ for women. Physical performance was assessed by the 5-times chair stand test [15]. The low physical performance diagnostic cut-off was $\geqq 12 \mathrm{~s}$ [9]. All patients were measured for muscle strength and physical performance using the same method by a physical therapist at each facility on dialysis day before dialysis treatment.

\section{Outcomes}

The primary study outcome was all-cause mortality. This outcome was assessed based on the death registry and medical records at the clinic.

\section{Statistics}

Data are expressed as mean \pm standard deviation (SD) or percentages, whenever appropriate. The relative risk of mortality for the muscle strength and physical performance as possible sarcopenia variables were estimated using Cox proportional hazard models with adjustment for age, sex, nutritional status, smoking status, dialysis efficiency, and comorbidities. Hazard ratios and their $95 \%$ confidence intervals (CI) were calculated using the estimated regression coefficients and their 
standard errors in Cox regression analysis. The survival curve was calculated by the Kaplan-Meier method. The $p$-value for comparison of the survival curve was determined by the log-rank test. The relationship between possible sarcopenia (handgrip strength, 5-times chair stand test) and nutritional disorder (GNRI) was constructed receiver operating characteristic (ROC) curves for outcomes using two variables. We used the Youden index to determine the optimal cut-off points for GNRI [16]. Youden index is used to measure the overall combined specificity and sensitivity of prognostic factor and is defined as the maximum vertical distance between the ROC curve and the diagonal of chance line and is calculated as maximum. The best Youden index is used to determine the best cut-off point of GNRI. $P$-value $<0.05$ was considered statistically significant. Statistical analyses were performed using SPSS (version 25, IBM, Tokyo).

\section{Results}

Patient demographics, nutritional status, and probable sarcopenia index

A total of 635 Japanese patients were analyzed. Table 1 shows the patients' demographics, nutritional status, and probable sarcopenia index.

\section{Kaplan-Meier estimate and cox proportional hazards regression analysis of patients' survival}

The median follow-up of patients was 1003 days, during which 62 deaths (10\%) were reported. Kaplan-Meier analysis revealed that patients with a handgrip and 5-times chair stand test below the possible sarcopenia cut-off had significantly lower survival rates than those with a higher function (log-rank test, $P<0.001$, Figs. 1 and 2).

Univariate cox proportional hazards analysis for mortality showed that the handgrip and 5-times chair stand test were significant predictors for mortality (Table 2).

Table 1 Baseline characteristics staratified by gender

\begin{tabular}{|c|c|c|}
\hline Variable & Male $(n=355)$ & Female $(n=280)$ \\
\hline \multicolumn{3}{|l|}{ Characteristicss } \\
\hline Age (y) & $70.5(10.7)$ & $68.6(10.3)$ \\
\hline Body mass index $\left(\mathrm{kg} / \mathrm{m}^{2}\right)$ & $22.2(3.38)$ & $21.7(4.26)$ \\
\hline Smoking (\%) & 10.4 & 1.8 \\
\hline Dialysis vintage $(m)$ & $86.5(84.3)$ & 87.6(85.3) \\
\hline \multicolumn{3}{|l|}{ ESRD primary cause (\%) } \\
\hline Diabetes Mellitus & 38.9 & 29.3 \\
\hline Nephrosclerosis & 16.9 & 15.0 \\
\hline Nephritis & 21.4 & 35.4 \\
\hline Other & 22.8 & 20.4 \\
\hline \multicolumn{3}{|l|}{ Comorbid conditions (\%) } \\
\hline Diabetes Mellitus & 33.4 & 27.6 \\
\hline Hypertension & 63.3 & 53.3 \\
\hline Hyperlipemia & 15.8 & 20.2 \\
\hline \multicolumn{3}{|l|}{ Laboratory and nutritional conditions } \\
\hline Serum albumin (g/dL) & $3.64(0.72)$ & $3.59(0.29)$ \\
\hline Serum hemoglobin (g/dL) & $11.8(0.96)$ & $10.9(0.95)$ \\
\hline Serum C-reactive protein (mg/dL) & $0.41(0.82)$ & $0.26(0.58)$ \\
\hline Serumn PTH intact $(p g / m L)$ & 159.8(96.2) & $160.7(105.8)$ \\
\hline $\mathrm{Kt} / \mathrm{N}$ & $1.48(0.26)$ & $1.76(0.31)$ \\
\hline Normalized protein catabolic rate & $1.09(0.22)$ & $0.89(0.19)$ \\
\hline GNRI & $92.4(6.88)$ & $91.5(6.69)$ \\
\hline GNRI<90 (\%) & 31.0 & 36.8 \\
\hline \multicolumn{3}{|l|}{ Physical Functioning } \\
\hline Handgrip (kg) & $25.5(8.02)$ & $17.7(5.57)$ \\
\hline Handgrip male<28kg, female<18kg (\%) & 62.5 & 52.9 \\
\hline 5-times chair stand test (sec.) & $11.8(4.50)$ & $11.4(4.51)$ \\
\hline 5-times chair stand test $\geqq 12$ seconds (\%) & 41.7 & 33.6 \\
\hline
\end{tabular}

ESRD End stage renal disease, PTH Parathyroid hormone, GNRI Geriatric nutritional risk index 


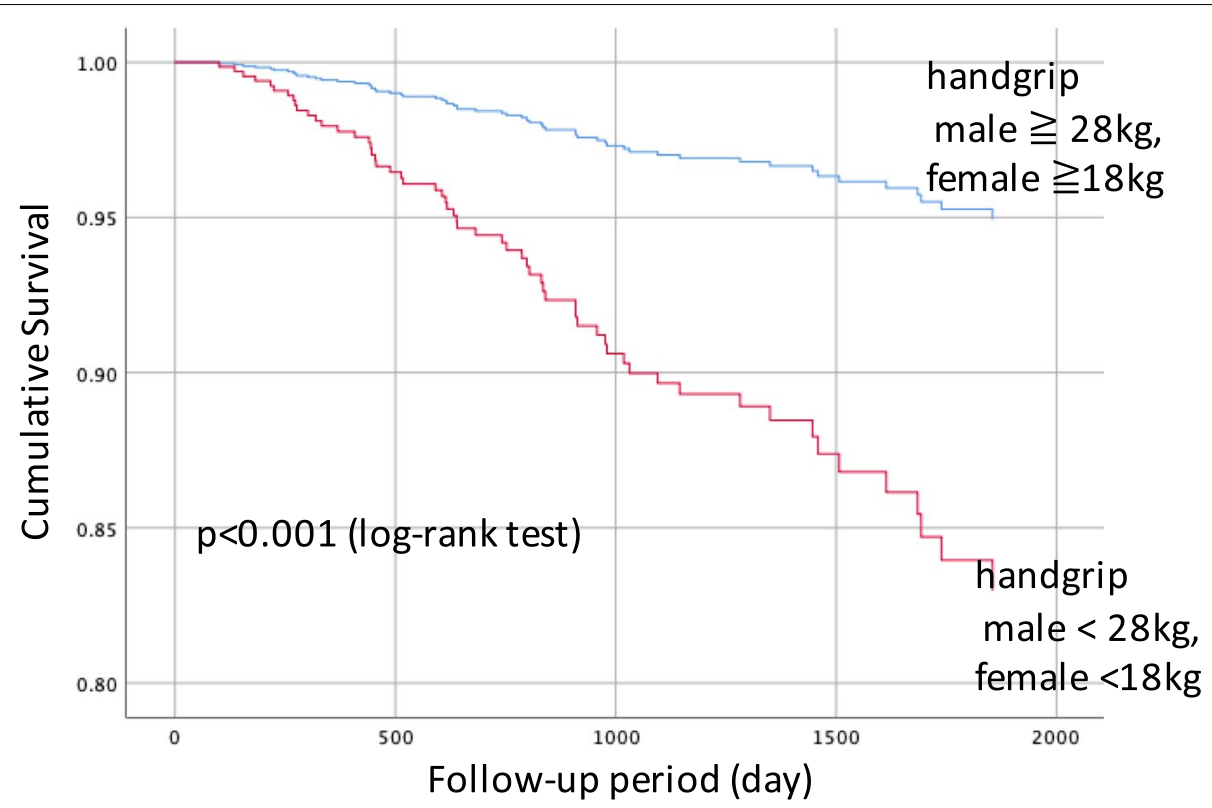

Fig. 1 Handgrip and survival of hemodialysis patients. Patients with under cut-off value handgrip significantly lower survival rate during the follow-up period, compared with upper cutoff value (Kaplan-Meier analysis)

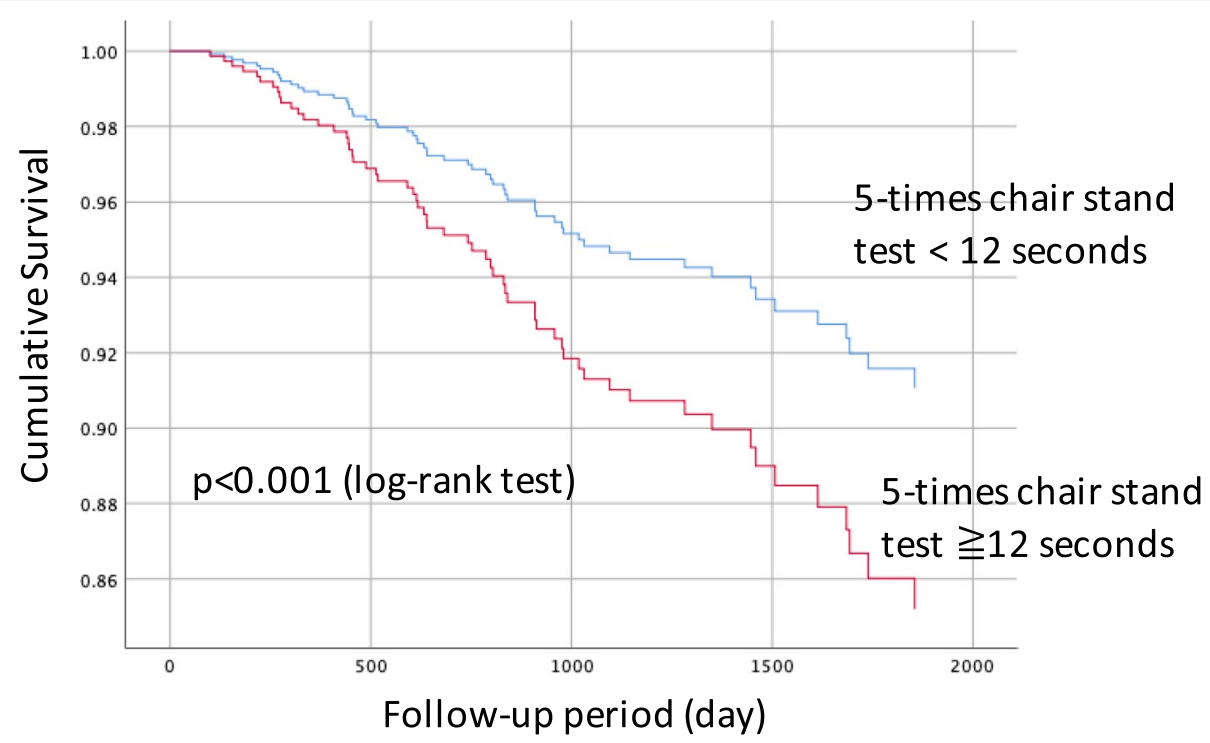

Fig. 25 -times chair stand test and survival of hemodialysis patients. Patients with 5 -times chair stand test $\geqq 12 \mathrm{~s}$ had a significantly lower survival rate during the follow-up period, compared with 5-times chair stand test $<12$ (Kaplan-Meier analysis)

Moreover, in the multivariate cox proportional hazards analysis, the handgrip (HR 3.61, 95\% CI 1.70-7.68, $p<0.001$ ) and the 5-times chair stand test (HR $1.7195 \%$ CI 1.01-2.90, $p=0.045)$ were significant predictors for mortality (Table 2).
Relationship between nutritional status and possible sarcopenia indices

ROC curve analysis was performed for the GNRI (Fig. 3). On the evaluation of possible sarcopenia by handgrip strength, the area under curve (AUC) on ROC curve analysis were 0.68 (95\% CI 0.64-0.72), and 5-chair stand, 
Table 2 Association between probable sarcopenia incices (handgrip, 5-times chair stand test) and mortality in cox proportional hazard regression models

\begin{tabular}{lll}
\hline & $\begin{array}{l}\text { Handgrip male<28kg, } \\
\text { female<18kg }\end{array}$ & $\begin{array}{l}\text { 5-time chair } \\
\text { stand test } \geqq \mathbf{1 2} \\
\text { seconds }\end{array}$ \\
\hline $\begin{array}{l}\text { Univariate Model } \\
\text { HR (95\% Cl) }\end{array}$ & $4.86(2.39-9.89)$ & $1.92(1.15-3.21)$ \\
$\quad \begin{array}{l}\text {-value } \\
\text { Multivariate Model }\end{array}$ & .000 & .013 \\
HR (95\% Cl) & $3.61(1.70-7.68)$ & $1.71(1.01-2.90)$ \\
P-value & .001 & .045 \\
\hline
\end{tabular}

Analyis were performing using Cox proportional hazards regression. Multivariate model included sex, age, normalized catabolic rate, $\mathrm{Kt} / \mathrm{V}$, diabetes mellitus, hypertension, hyperlipemia, smking

HR Hazard ratio, Cl Confidence interval, GNRI Geriatirc nutritional risk index

the AUC on ROC were 0.55 (95\% CI 0.51-0.60). The cutoff value for the GNRI discriminating those at possible sarcopenia by handgrip strength based on the Youden index was 91.5 (Fig. 4).

\section{Discussion}

In this prospective cohort and observational study, we clarified that handgrip and the 5-times chair stand test, indicated by the new sarcopenia consensus, the AWGS 2019, as a prognostic indicator for dialysis patients, are useful screening tools. In particular, handgrip strength was more than twice as high as the hazard risk compared to the 5-times chair stand test, which may be useful in terms of predicting mortality risk by assessing possible sarcopenia.

The relationship between reduced handgrip and mortality for dialysis patients has been previously reported [17] [18]. However, Vogt's study differs from the conditions in our study in that the subjects' were younger (as young as 50 years of age), and the cut-off value for handgrip reduction was $22.5 \mathrm{~kg}$ for males and $7 \mathrm{~kg}$ for females [17]. The finding of a significantly higher correlation between handgrip and mortality using the revised AWGS 2019 sarcopenia consensus appears to be a novel outcome. Furthermore, it is considered that in the results of our study, handgrip has been shown to correlate with systemic skeletal muscle function and malnutrition, which is linked to the results of this study suggest that handgrip was more strongly associated with mortality than the 5-times chair stand test [19-22].

The second finding of this study was that handgrip, a measure of possible sarcopenia, was significantly correlated with GNRI. The cut-off value for estimating handgrip strength loss for possible sarcopenia was 915. In a previous study of Japanese dialysis patients, the cut-off value for GNRI was reported to be 91-92 when the outcome setting was death $[5,6]$. Our study of the cut-off value for probable sarcopenia was similar, suggesting that the risks of malnutrition, probable sarcopenia, and death are interrelated in the pathogenesis of PEW. Moreover,
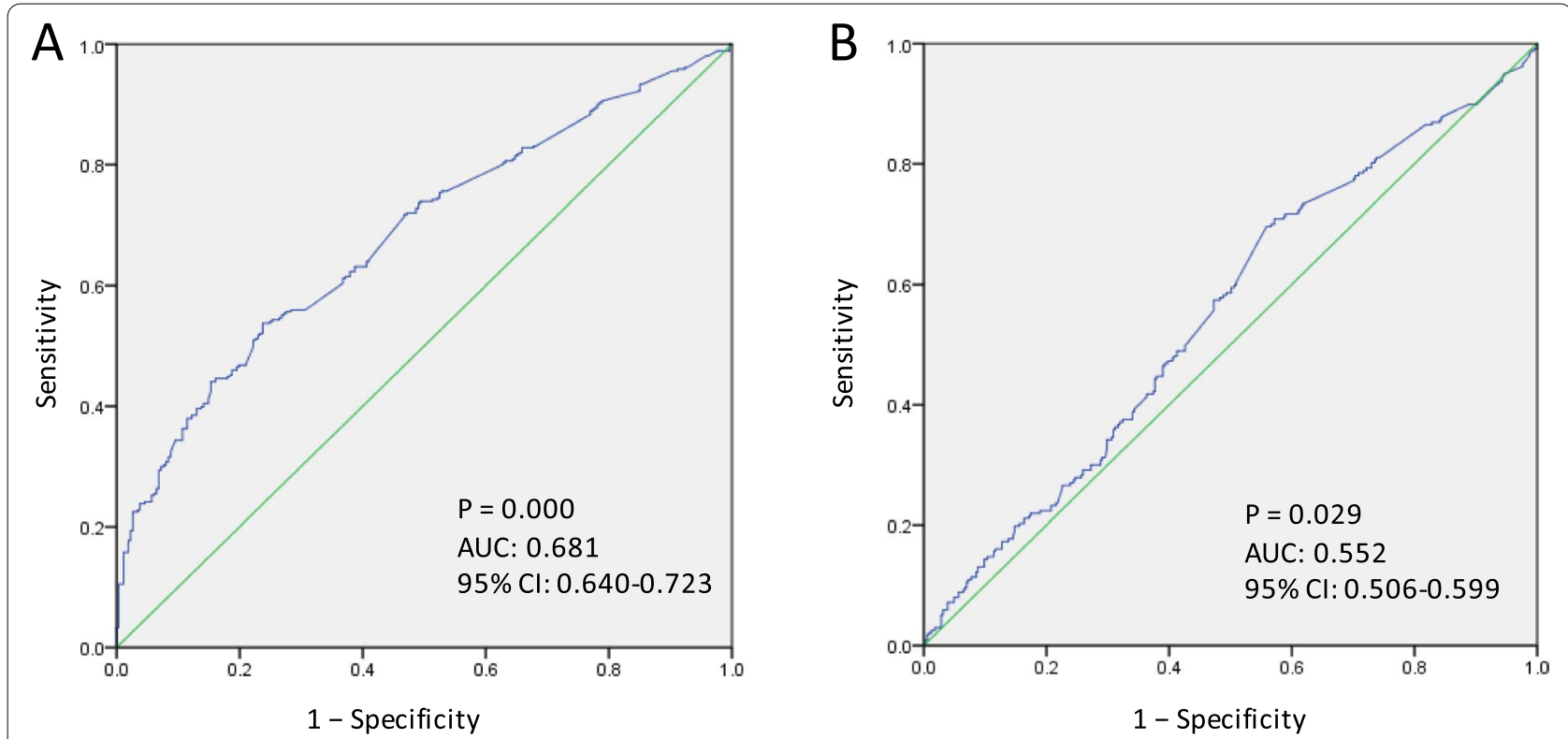

Fig. 3 Receiver operating characteristics curves of GNRI for possible sarcopenia. Receiver operating characteristics curves of GNRI for possible sarcopenia by handgrip strength $(\mathbf{A})$ and possible sarcopenia by 5-times chair stand test (B). In both (A) and (B), the AUC was significant, and (A) having a larger AUC. AUC, area under the curve; Cl, confidence interval. 


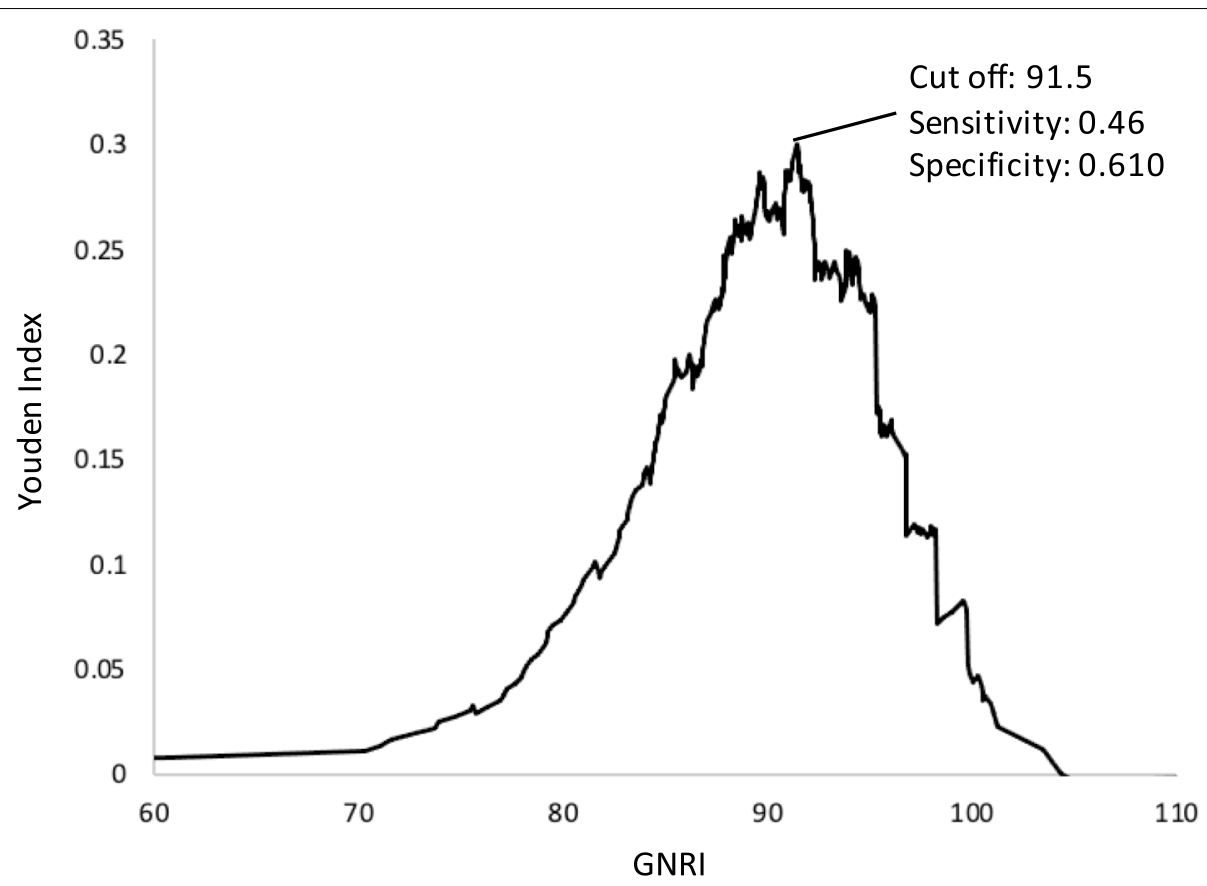

Fig. 4 The cut-off value of GNRI detected by the Youden Index. GNRI, geriatric nutritional risk index

several studies on the usefulness of the GNRI in dialysis patients have shown that the GNRI has a higher sensitivity and specificity than other assessment tools for predicting mortality [23] [24]. Therefore, GNRI may be useful as a screening index based on medical record information as a substitute for SARC-F as described in the consensus [8] in estimating sarcopenia.

Another noteworthy aspect of this study is that the mortality rate during the observation period was $10 \%$, which was a little lower than that of other country studies $[11,25]$, despite the large number of patients with low BMI. The reason for this may be that Japan's universal health insurance system allows all dialysis patients to receive early treatment for complications, and it should be recognized that many patients may be thin because of differences in eating habits and not many PEW patients.

There were several limitations to our study. First, we have not been able to assess muscle mass, which is essential for sarcopenia diagnosis and can only refer to the relationship between the possible sarcopenia and mortality. Second, although we adjusted for comorbidities and other factors, this was an observational study, so interventional studies are needed to clarify the relationship between sarcopenia and death. Finally, although the GNRI cut-off value was presented as screening before possible sarcopenia assessment, the serum albumin level was not adjusted for diet or dialysis dose.

In conclusion, our study suggests that the handgrip strength test of the AWGS 2019 sarcopenia consensus (cut-off value, male $<28 \mathrm{~kg}$, female $<18 \mathrm{~kg}$ ) was the simple and useful tool to predict mortality in chronic hemodialysis patients. Furthermore, GNRI assessment can be a useful tool for screening before assessing possible sarcopenias, such as muscle strength and exercise performance, when it is difficult to perform SARC-F for all patients.

\section{Abbreviations}

PEW: Protein-energy wasting syndrome; AWGS: Asian Working Group for Sarcopenia;; GNRI: Geriatric Nutritional Risk Index; SPPB: Short Physical Performance Battery; HR: Hazard ratio; 95\% Cl: 95\% confidence interval; BMI: Body mass index; Kt $N$ : Standardized dialysis volume; nPCR: Normalized protein catabolic rate; SD: Standard deviation; Cl: Confidence Interval; OR: Odds Ratio.

\section{Acknowledgments}

The authors thank the renal staff for their support and the patients for giving their time to complete the research protocol.

\section{Authors' contributions}

$\mathrm{KK}, \mathrm{YM}, \mathrm{HY}, \mathrm{AH}, \mathrm{TI}, \mathrm{TY}$, and YN contributed to the conception and design of the work, the acquisition, analysis, and interpretation of data, drafting or revision of the manuscript, and final approval of the version to be submitted.

\section{Funding}

This work was supported by grants from the JSPS KAKENHI [Grant Number $17 \mathrm{~K} 13097]$. The funding source had no role in the design, data collection, analysis, and study findings.

\section{Availability of data and materials}

The datasets used and/or analyzed during the current study are available from the corresponding author on reasonable request. 


\section{Declarations}

\section{Ethics approval and consent to participate}

The study protocol complied with the Helsinki Declaration standards and was approved by the Ethical Committee of the International University of Health and Welfare (Approval number 17-lo-95). The requirement for written informed consent was waived as this study used retrospective data.

\section{Consent for publication}

Not Applicable.

\section{Competing interests}

The authors declare that they have no competing interests.

\section{Author details}

${ }^{1}$ Department of Physical Therapy, International University of Health and Welfare Graduate School, -3 Kozunomori, Narita, Chiba 286-8686, Japan. ${ }^{2}$ Department of Rehabilitation, Kaikoukai Nagoya Kyoritsu Hospital, Nagoya, Aichi, Japan. ${ }^{3}$ Department of Physical Therapy, Seirei Christopher University. Schoo of Rehabilitation, Hamamatsu, Shizuoka, Japan. ${ }^{4}$ Dialysis division at Shizuoka, Kaishokai, Shizuoka, Shizuoka, Japan. ${ }^{5}$ Dialysis division, Kaikoukai Healthcare Group, Nagoya, Aichi, Japan.

Received: 11 June 2020 Accepted: 13 October 2021

Published online: 12 November 2021

\section{References}

1. Carrero JJ, Stenvinkel P, Cuppari L, Ikizler TA, Kalantar-Zadeh K, Kaysen G, et al. Etiology of the protein-energy wasting syndrome in chronic kidney disease: a consensus statement from the International Society of Renal Nutrition and Metabolism (ISRNM). J Ren Nutr. 2013;23(2):77-90.

2. Isoyama N, Qureshi AR, Avesani CM, Lindholm B, Bárány P, Heimbürger O, et al. Comparative associations of muscle mass and muscle strength with mortality in dialysis patients. Clin J Am Soc Nephrol. 2014;9(10):1720-8.

3. Domaski M, Ciechanowski K. Sarcopenia: a major challenge in elderly patients with end-stage renal disease. J Aging Res. 2012;2012(1):754739.

4. Cruz-Jentoft AJ, Sayer AA. Sarcopenia. Lancet. 2019;393(10191):2636-46 Available from: https://linkinghub.elsevier.com/retrieve/pii/S014067361 9311389.

5. Zelle DM, Klaassen G, van Adrichem E, Bakker SJL, Corpeleijn E, Navis G. Physical inactivity: a risk factor and target for intervention in renal care. Nat Rev Nephrol. 2017;13(3):152-68 Available from: http://www.nature. com/articles/nrneph.2016.187.

6. Wang T, Feng X, Zhou J, Gong H, Xia S, Wei Q, et al. Type 2 diabetes mellitus is associated with increased risks of sarcopenia and pre-sarcopenia in Chinese elderly. Sci Rep. 2016;6:1-7.

7. Cui M, Gang X, Wang G, Xiao X, Li Z, Jiang Z, et al. A cross-sectional study. Medicine (Baltimore). 2020;99(2):e18708 Available from: https://journals. Iww.com/10.1097/MD.0000000000018708.

8. Chen L-K, Woo J, Assantachai P, Auyeung T-W, Chou M-Y, lijima K, et al. Asian working group for sarcopenia: 2019 consensus update on sarcopenia diagnosis and treatment. J Am Med Dir Assoc. 2020;21(3):300-307.e2 Available from: https://linkinghub.elsevier.com/retrieve/pii/S152586101 9308722

9. Kobayashi I, Ishimura E, Kato Y, Okuno S, Yamamoto T, Yamakawa T, et al. Geriatric nutritional risk index, a simplified nutritional screening index, is a significant predictor of mortality in chronic dialysis patients. Nephrol Dial Transplant. 2010;25(10):3361-5.

10. Yamada K, Furuya R, Takita T, Maruyama Y, Yamaguchi Y, Ohkawa S, et al. Simplified nutritional screening tools for patients on maintenance hemodialysis. Am J Clin Nutr. 2008;87(1):106-13.
11. Fukuma S, Ikenoue T, Akizawa T, Fukuhara S. Impact of nutritional index on the association between phosphorus concentrations and mortality in haemodialysis patients: a cohort study from dialysis outcomes and practice pattern study in Japan. BMJ Open. 2017;7(8):1-9.

12. Kono K, Nishida Y, Yabe H, Moriyama Y, Mori T, Shiraki R, et al. Development and validation of a fall risk assessment index for dialysis patients. Clin Exp Nephrol. 2018:22(1):167-72.

13. Bouillanne O, Morineau G, Dupant C, Coulombel I, Vincent JP, Nicolis I, et al. Geriatric nutritional risk index: a new index for evaluating at-risk elderly medical patients. Am J Clin Nutr. 2005;82(4):777-83.

14. Okamoto T, Hatakeyama S, Kodama H, Horiguchi H, Kubota Y, Kido K, et al. The relationship between poor nutritional status and progression of aortic calcification in patients on maintenance hemodialysis. BMC Nephrol. 2018;19(1):1-8

15. Guralnik JM, Simonsick EM, Ferrucci L, Glynn RJ, Berkman LF, Blazer DG, et al. A short physical performance battery assessing lower extremity function: association with self-reported disability and prediction of mortality and nursing home admission. J Gerontol. 1994;49(2):85-94.

16. Matsuzawa R, Roshanravan B, Shimoda T, Mamorita N, Yoneki K, Harada $M$, et al. Physical activity dose for hemodialysis patients: where to begin? Results from a prospective cohort study. J Ren Nutr. 2018;28(1):45-53. Available from. https://doi.org/10.1053/j.jrn.2017.07.004.

17. Vogt BP, Borges MCC, de Goés CR, Caramori JCT. Handgrip strength is an independent predictor of all-cause mortality in maintenance dialysis patients. Clin Nutr. 2016;35(6):1429-33.

18. Matos CM, Silva LF, Santana LD, Santos LS, Protásio BM, Rocha MT, et al. Handgrip strength at baseline and mortality risk in a cohort of women and men on hemodialysis: A 4-year study. J Ren Nutr. 2014;24(3):157-62 [cited 2020 Mar 12]. Available from: https://linkinghub.elsevier.com/retri eve/pii/S1051227613002288.

19. Heimbürger O, Qureshi AR, Blaner WS, Berglund L, Stenvinkel P. Hand-grip muscle strength, lean body mass, and plasma proteins as markers of nutritional status in patients with chronic renal failure close to start of dialysis therapy. Am J Kidney Dis. 2000;36(6):1213-25.

20. Wang AYM, Sea MMM, Ho ZSY, Lui SF, Li PKT, Woo J. Evaluation of handgrip strength as a nutritional marker and prognostic indicator in peritoneal dialysis patients. Am J Clin Nutr. 2005;81(1):79-86.

21. Leal VO, Mafra D, Fouque $D$, Anjos LA. Use of handgrip strength in the assessment of the muscle function of chronic kidney disease patients on dialysis: a systematic review. Nephrol Dial Transplant. 2011;26(4):1354-60.

22. Leal VO, Stockler-Pinto MB, Farage NE, Aranha LN, Fouque D, Anjos LA, et al. Handgrip strength and its dialysis determinants in hemodialysis patients. Nutrition. 2011;27(11-12):1125-9. Available from:. https://doi. org/10.1016/j.nut.2010.12.012.

23. Kalantar-Zadeh K, Kopple JD, Block G, Humphreys MH. A malnutritioninflammation score is correlated with morbidity and mortality in maintenance hemodialysis patients. Am J Kidney Dis. 2001;38(6):1251-63 [cited 2020 Mar 13]. Available from: https://www.sciencedirect.com/science/ article/abs/pii/S0272638601780602?via\%3Dihub.

24. Beberashvili I, Azar A, Sinuani I, Shapiro G, Feldman L, Sandbank J, et al. Geriatric nutritional risk index, muscle function, quality of life and clinical outcome in hemodialysis patients. Clin Nutr. 2016;35:1522-9.

25. Roshanravan B, Robinson-Cohen C, Patel KV, Ayers E, Littman AJ, De Boer $\mathrm{IH}$, et al. Association between physical performance and all-cause mortality in CKD. J Am Soc Nephrol. 2013;24(5):822-30.

\section{Publisher's Note}

Springer Nature remains neutral with regard to jurisdictional claims in published maps and institutional affiliations. 